\title{
Úlceras por presión en las unidades de cuidados intensivos: ¿inevitables o prevenibles?
}

\section{Can besores be avoided in intensiva care units?}

\author{
Francisco Manzano Manzano ${ }^{a, *}$ y Carmen Corral Rubio ${ }^{b}$
}

\begin{abstract}
a Médico intensivista, Servicio de Cuidados Críticos, Hospital Universitario Virgen de las Nieves, Granada, España
bProfesora de la Escuela de Enfermería, Hospital Universitario Virgen de las Nieves, Granada, España
\end{abstract}

La incidencia de las úlceras por presión (UPP) en las unidades de cuidados intensivos (UCI) continúa siendo elevada. A pesar de la cantidad de recursos invertidos, continuamos sufriendo este grave problema de salud. El trabajo del Dr. Yepes y colaboradores en 150 pacientes de una $\mathrm{UCl}$ en Bolivia indica un $26,7 \%$ de incidencia de UPP de grado II 0 superior $^{1}$. Esta elevada cifra es recientemente confirmada por otros autores, como Nijs y colaboradores ${ }^{2}$, que en 2008 publican una incidencia del 20,1\%de UPP grado II o superior en una muestra de 520 enfermos de una UCI médico-quirúrgica. En la última década se han publicado estudios epidemiológicos con resultados contradictorios, aunque mayoritariamente las incidencias señaladas son altas, con cifras que oscilan entre el 1,9\% el 29,5\% Las discrepancias entre estas cifras pueden ser consecuencia de la comparación entre muestras muy diferentes, y es evidente que el paciente crítico presenta una mayor susceptibilidad al desarrollo de UPP debido a la frecuente inmovilidad y a la exposición a múltiples factores de riesgo asociados con la enfermedad, tales como edad elevada, prolongadas estancias en $\mathrm{UCl}$, alteraciones hemodinámicas y del intercambio gaseoso, etc. Ante estas elevadas cifras, la reducción de la incidencia es un objetivo prioritario. Se han recomendado actuaciones como la evaluación del riesgo de UPP, maniobras para redistribuir la presión, intervenciones dirigidas a prevenir o reducir la exposición a la fricción o el cizallamiento, inter-

\footnotetext{
*Autor para correspondencia

Correo electrónico: fmanzano@mailpersonal.com (F. Manzano Manzano).
}

venciones dirigidas a mitigar factores contribuyentes tales como la humedad o los trastornos de la nutrición y, finalmente, la educación a los pacientes, las familias y los proveedores de cuidados ${ }^{3}$. A pesar de la implantación de las normativas de supervisión y de las mejoras en las tecnologías disponibles para la prevención, los datos epidemiológicos demuestran una estabilidad en la incidencia de las UPP. Por ello una de las cuestiones que se plantean es si el fracaso en la reducción de la incidencia de UPP se relaciona primordialmente con los factores asociados con la morbilidad de los pacientes o bien con factores que dependen de los cuidadores y que, por tanto, están relacionados con la implementación o la calidad de las medidas preventivas aplicadas. De aquí se derivan dos visiones completamente distintas de este problema. Por un lado, una visión que defendería que las UPP son inevitables, aunque podría reducirse la incidencia a niveles más bajos que los actuales si se incorporasen nuevos avances tecnológicos o científicos, y otra que consideraría que son prevenibles y que se podría alcanzar la incidencia cero si se consiguiera la excelencia en la aplicación de las medidas preventivas. Con la información disponible hasta ahora, no es posible saber cuál de estas posiciones se halla más próxima a la realidad, ya que la mayor parte de la producción científica relacionada con Ias UPP se centra en los factores o escalas de evaluación del riesgo de UPP, pero escasamente se menciona el impacto de la calidad de los cuidados del personal sanitario a los pacientes. Sin embargo, este factor no es descartable que tenga un papel decisivo en la ocurrencia de las UPP en las UCl. Así, también nos preguntamos: ¿qué porcentaje de las UPP puede ser evitable? Por el momento no disponemos de 
estudios científicos que puedan responder a esta pregunta, pero basándonos en nuestra experiencia clínica pensamos que probablemente incidencias menores del $7 \%$ en enfermos ventilados podrían ser razonables si se aplicasen unos cuidados ideales. En consecuencia, podemos decir que, con pocas excepciones, las UPP deberían ser inaceptables.

Los profesionales de la salud hemos caído en la trampa de aceptar la inevitabilidad de las UPP, y mientras no cambiemos nuestra manera de pensar, y nos acerquemos a la cuestión de la aparición de las UPP como un problema de calidad de los cuidados, nada va a cambiar y no vamos a conseguir una reducción importante de esta lacra en las $\mathrm{UCl}$. No podemos aceptar niveles de calidad mediocres al ocuparnos de las UPP en las UCl en comparación con otros ámbitos de la enfermería tales como el control del riesgo de infección, la administración de la medicación o la seguridad del paciente, donde los niveles de exigencia son mayores. Es muy preocupante que muchos enfermos en las UCI desarrollen UPP, debido a que los profesionales de la salud estamos muy centrados en tratar la disfunción de órgano que presenta el enfermo, que tiene un mayor impacto sobre la morbimortalidad, pero nos olvidamos de los cuidados de la piel, que deberían ser también siempre una prioridad.

En el proceso de la prevención de las UPP, uno de los principales retos que se plantean en el futuro de las $\mathrm{UCl}$ es conocer cuál es la incidencia y las actuaciones preventivas aplicadas en una población de enfermos cuyas características homogéneas y fácilmente reproducibles permitan la comparación entre diferentes unidades y centros hospitalarios. Este hecho es apoyado por el fracaso de las escalas de riesgo de UPP (Braden, Norton, etc.) que, utilizadas en la población general hospitalaria, han demostrado escasa capacidad discriminatoria en enfermos críticos. Dentro de la heterogeneidad de las poblaciones de enfermos que ingre- san en las $\mathrm{UCl}$, la mej or población que reúne estas características es la de enfermos con fallo respiratorio agudo, o sea, que precisan ventilación mecánica durante un periodo mayor de 24 horas, enfermos que además presentan una alta incidencia de UPP. En el artículo de Yepes y colaboradores $^{1}$, el $89 \%$ de los enfermos precisa de ventilación mecánica, pero no se indica cuál es la incidencia de UPP en este subgrupo de enfermos, aunque probablemente sea más alta que en el resto de la población general de la $\mathrm{UCl}$. De igual manera, la mayoría de los trabajos que estudian las UPP en UCI tampoco aporta datos sobre la incidencia de UPP en estos enfermos, por lo que no sabemos si las diferencias en las incidencias señaladas son consecuencia de las distintas poblaciones estudiadas o bien de diferencias en las estrategias preventivas. Por ello sería deseable hallar en el futuro publicaciones científicas que indiquen tanto la incidencia bruta en UCl como la incidencia de UPP en este subgrupo de enfermos.

En suma, todavía necesitamos mejorar nuestros conocimientos, actitudes y estrategias preventivas respecto a las UPP en $\mathrm{UCl}$, para poder afirmar que es posible la incidencia cero.

\section{Bibliografía}

1. Yepes D, Molina F, León W, Pérez E. Incidencia y factores de riesgo asociados a la presencia de úlceras por presión en enfermos críticos. Med Intensiva. 2009;33:272-7.

2. Nijs N, Toppets A, Defloor T, Bernaerts K, Milisen K, Van Den Berghe $G$. Incidence and risk factors for pressure ulcers in the intensive care unit. J Clin Nurs 2008; dec 1-9 [Epub ahead of print].

3. Reddy M, Gill SS, Rochon PA. Preventing pressure ulcers: a systematic review. J AMA. 2006;296:974-84. 